University of San Diego

Digital USD

Spring 5-23-2020

\title{
Bridging the Gap: Improving Hospital Discharge through Utilization of a Priority Care Clinic
}

Kristian Jamerson

University of San Diego, kjamerson@sandiego.edu

Follow this and additional works at: https://digital.sandiego.edu/dnp

Part of the Family Practice Nursing Commons

\section{Digital USD Citation}

Jamerson, Kristian, "Bridging the Gap: Improving Hospital Discharge through Utilization of a Priority Care Clinic" (2020). Doctor of Nursing Practice Final Manuscripts. 118.

https://digital.sandiego.edu/dnp/118

This Doctor of Nursing Practice Final Manuscript is brought to you for free and open access by the Theses and Dissertations at Digital USD. It has been accepted for inclusion in Doctor of Nursing Practice Final Manuscripts by an authorized administrator of Digital USD. For more information, please contact digital@sandiego.edu. 
UNIVERSITY OF SAN DIEGO

Hahn School of Nursing and Health Science

DOCTOR OF NURSING PRACTICE

BRIDGING THE GAP: IMPROVING HOSPITAL DISCHARGE THROUGH

UTILIZATION OF A PRIORITY CARE CLINIC

by

Kristian Jamerson

A Doctor of Nursing Practice Portfolio presented to the

FACULTY OF THE HAHN SCHOOL OF NURSING AND HEALTH SCIENCE

UNIVERSITY OF SAN DIEGO

In partial fulfillment of the

requirements for the degree

DOCTOR OF NURSING PRACTICE

May 2020 


\begin{abstract}
Background: Poorly coordinated care often results in poor health outcomes. Best practices in academic literature recommends effective programs that consist of comprehensive discharge planning, complete and timely communication of information, medication reconciliation, patient and caregiver education using the teach-back method, open communication among providers, and prompt follow-up visits with an outpatient provider after discharge. Using a priority care clinic (PCC) for follow-up is one approach to decrease hospital readmissions.
\end{abstract}

Purpose: To implement an evidence-based program utilizing a PCC to facilitate post discharge transition-in-care and reduce hospital readmissions.

Evidence-based Intervention: A PCC assists patients in their transition of care from the hospital to home. Delays in follow-up appointments and lapse in medication adherence may occur between hospital discharge and the first follow-up visit, thus resulting in unnecessary hospital readmissions.

Evaluation/Results: Data will be retrospectively collected and reviewed from patients' charts to assess for compliance with (a) follow-up visit appointment, (b) medication adherence, and (c) readmission rates using a nurse practitioner led follow-up phone call and comparing results to the national benchmark.

Implication for Practice: PCCs are the necessary bridge to facilitate increased compliance with prompt follow-up visits and medication reconciliation to ensure adherence. 
Conclusion: Implementation of a PCC addresses the need for reducing avoidable readmissions and complying with reimbursement standards required by the Centers for Medicare and Medicaid Services while meeting the best practice needs for patients. 
Bridging the Gap: Improving Hospital Discharge through

Utilization of a Priority Care Clinic

\section{Description of the Clinical Problem}

Researchers indicate that comprehensive discharge planning, complete and timely communication of information, medication reconciliation, education using the teach-back method, open communication between providers, and prompt follow-up visits with an outpatient provider after discharge are the qualities of a best practice program (Jackson et al., 2015). Poorly coordinated care transitions often result in poor health outcomes; lack of supportive post-discharge resources has resulted in frequent hospital readmissions costing an estimated $\$ 44$ billion per year (Dreyer, 2014). Reimbursement changes under the Affordable Care Act have obligated providers to search for solutions to improve transitions in care in order to receive incentives from the Center for Medicare and Medicaid Services (CMS). Timely outpatient follow-up is promoted as key module of transitional care to reduce hospital readmissions. Fragmented health systems throughout the world have led to unmet health needs. Interprofessional health care teams can address this concern by optimizing the skills of their members to provide better health services to patients resulting in strengthen health outcomes (Gilbert et al., 2010)

\section{Description of EBP Project, Facilitators, and Barriers}

The aim of this evidence-based practice (EBP) project was to increase the use of a priority care clinic (PCC) to facilitate post discharge transition in care needs by offering medication reconciliation, specialty referrals, and provider contact within 7 days of discharge to reduce hospital readmissions. The Director of Outreach and Priority Care Services assisted with the standardization of education for staff members on use of the 
PCC. Desert Oasis implemented the PCC to meet their patients' needs post hospital discharge to reduce hospital readmissions. The goal was to educate staff members on appropriate referrals to the PCC to ensure patients were accessing this free resource. A formal in-service training was conducted to educate frontline staff members on use of the PCC, resources available for patients, and timely referrals. Case managers followed guideline criteria for PCC enrollment. A warm handoff between case managers and the post utilization team entailed patient descriptive information, diagnosis, and date of discharge to determine PCC eligibility as well as appointment retrieval prior to the patient's hospital discharge.

Facilitators of this program included the support of case managers, social workers, primary care providers, medical assistants, ancillary staff members, and patients. Barriers included patient buy-in; some patients were not comfortable seeing a different provider and chose to wait for an appointment with their primary care provider. Residences in the Yucca Valley area require distance travel from the hospital as well as the PCC; transportation was a barrier for some patients. Desert Oasis partnered with a local transportation company to offer round trip rides to appointments to help mitigate this problem. Lack of timely communication between case managers and post utilization team members due to limited staff members was another barrier that hindered synchronization of patient participation and a plan of care prior to discharge to reduce hospital readmissions.

\section{Evidence-Based Project Model}

The Johns Hopkins model assisted in the development of a clinical decisionmaking and problem-solving tool that could be implemented by a nurse practitioner to 
ensure the latest research findings and best practices were used for patient care. The model consisted of a three-step process, called PET, to address the practice question, evidence, and translation (Dang \& Dearholt, 2018). The John Hopkins Model was used to assist with the piloting the PCC project to bring awareness to best practices, which included medication reconciliation and transition in care. PCC aligned with the goal of the John Hopkins model and offered a solution to meet the patients' needs and reduce hospital readmissions.

\section{Proposed Evidence-Based Solutions}

A review of the literature was completed with the following search engines: Cochrane, OVID, PubMed, Google Scholar, and CINAHL. Keywords utilized with each search engine were readmission, outpatient follow-up, and care transitions. This search yielded 598 articles from the past 5 years from peer-reviewed publications. To narrow the search further, the implementation of medical subject headings (MESH) terms included post-discharge and medication reconciliation. A total of 3 articles were chosen to validate the proposed interventions based on the applicability to hospital readmissions and best practice for transition in care. Each article was ranked using the Johns Hopkins' evidence level and quality guide tool (Dang \& Dearholt, 2018).

A quasi-experimental study with a level 2 ranking was accessed to analyze meaningful benefits from early outpatient follow-up. Jackson et al. (2015) reported transitional care resources were most beneficial to high risk patients who received followup within 7 days. The study focused on readmissions, defined as a return to any hospital within 30 days of a previous discharge for any reason other than childbirth. Outpatient follow-up was defined as an office visit with any primary care provider or a federally 
qualified health center with a current procedural terminology code for evaluation. The sample size of this study was 44,473 Medicaid recipients with 65,085 qualifying discharges. Results suggested that early follow-up after hospital discharge was variable and depended upon the patient's clinical complexity. The 7-day follow-up among patients with highest clinical complexity and underlying risk of readmission benefited from early follow-up when compared to patients with only one acute or chronic condition who did not benefit from early follow-up (Jackson et al., 2015).

Low et al. (2017) performed a systematic review of randomized controlled trials with a level 1 ranking and reported a significant reduction in 30-day readmissions and emergency department visits with the use of transitional care. A multidisciplinary team of junior physicians, home care nurses, physiotherapist, occupational therapist, speech therapist, pharmacist, medical social worker, and administrators formed an integrated practice unit. All team members had access to patient records and shared a common mission to reduce avoidable readmissions. A pre-hospital-discharge phase was implemented to determine patient eligibility. Comprehensive assessment and nursing needs were performed within 2 weeks of discharge along with medication reconciliation and patient caregiver education using standardized action plans and videos. Results indicated a reduction in hospital readmissions and emergency department visits, concluding that transitional care interventions should be used for patients who are at sufficiently high risk (Low et al., n.d., 2017).

Using survey instruments, Graham et al. ( 2018) sought to determine whether hospital readmissions were preventable. The team determined that hospitals were more likely to be successful at preventing readmissions within the first week after discharge. 
Late readmissions typically related to inadequate monitoring and inability to keep postdischarge follow-up appointments with primary care providers (Graham et al., 2018). Baldwin's (2016) quality improvement project consisted of physicians, nurses, pharmacists, case managers, and support staff evaluating a primary care-based multidisciplinary follow-up program. The program identified individuals at risk for hospital readmission suggested attention to the primary care practice structure for transition from hospitalization to outpatient settings in order to reduce readmissions.

In a centrally-randomized, single-center, pragmatic controlled trial, Thygesen et al. (2015) compared usual care to a home-visit intervention by a general practitioner and municipal nurse within 7 days of discharge. The trial focused on the medication rehabilitation plan, functional level, and need for health care initiatives. Although there was no statistically-significant difference in readmission and mortality between groups, there was evidence that a lower proportion of home-visit patients were readmitted within 30 days when compared with the control group (20.1\% versus $23.4 \%$; Thygesen et al., 2015).

Lastly; a quasi-experimental, prospective, level 1 study evaluated the effectiveness of a post hospital interprofessional care team visits on improved care transitions and decreased hospital readmission rates. A discharge clinic was utilized for follow-up with patient post hospital discharge. Interprofessional care teams huddled to discuss a patient's recent hospitalization and developed an individualized care plan for the visit. Medical assistants performed necessary point-of-care testing and the nurse practitioner completed a thorough review of systems and a physical examination. Results showed a $2.7 \%$ readmission rate when compared to the national benchmark, 30-day 
readmission rate of $13.9 \%$ (Baldwin, 2016). A limitations in this study was the exclusion of patients who were bedbound or lacked transportation to the clinic. Additionally, inpatient staff had to be utilized due to lack of resources and employee availability on weekends and after hours.

\section{Program Development and Implementation Timeline}

For this project, Phase I included a meeting with the post utilization team members and ancillary staff to determine a need to increase PCC utilization, standardize PCC admission criteria, and verify medication reconciliation. In Phase II, a standardize education plan was developed and an in-service was hosted for case managers, medical assistants, and providers. The project was submitted to IRB for approval prior to implementation. The data did not suggest a reduction of no-show appointments for PCC; however, $95 \%$ of charts documented medication reconciliation and there was an overall reduction in hospital readmissions. The percent of charts with medication reconciliations exceeded previous weeks; post implementation, $100 \%$ of charts included medication reconciliations for several weeks. Statistical significance could not be demonstrated for

process indicator, PCC no-show appointments, as the clinic averaged merely 2 no-shows weekly, both pre-and post-implementation. Nonetheless, emergency department readmissions decreased from 1,871 to 439 during the year 2019.

\section{Data Analysis}

Analysis of the data revealed an increase of medication reconciliations exceeding 95\% weekly. While no-show appointments did not go down significantly, the number of no-shows averaged 2 per week. Lastly, there were no hospital readmissions established 
implementing the PCC and emergency department visits, in excess of 1,871 pre-PCC, were reduced to 439 visits.

\section{Organizational Gap Analysis of Project Site}

A retrospective review of data obtained through dashboards revealed a need to increase utilization of the PCC, sustain high rates of medication reconciliation, and standardize referrals methods. Educating providers and staff on the purpose and success of PCC referrals should help increase utilization of the PCC.

\section{Setting}

This project was conducted in a rural clinic in Southern California. Gaps in care were identified by one of the providers. The clinic included an urgent care for acute medical attention needs. A retrospective review of no-show, cancelled, and kept appointments were done for the months of August through October 2019 to determine PCC utilization. Medication reconciliation was included in the retrospective review.

\section{Ethical Issues}

IRB approval was obtained prior to the initiation of the PCC project. All patient information was de-identified to protect patients' privacy and be in compliance with the Health Insurance Portability and Accountability Act (HIPAA).

\section{Evidence-Based Intervention}

Standardizing education amongst staff members was intended to increase utilization of the PCC. A multidisciplinary approach was initiated to assist patients in their transition of care from the hospital to home. During staff in-service meetings, case managers were educated on admission criteria to PCC and appropriate specialty referrals for patients with a new diagnosis. The post-utilization team reviewed all referrals to the 
PCC daily and determined additional resources needed for each patient. An email was sent to the medical assistants at the PCC daily. Appointments were created for each patient, a medication reconciliation was completed at each visit, and specialty referral appointments were scheduled. A clinical provider assessed, diagnosed, and adjusted the treatment plan for each patient. Lastly, the provider educated the patient on new medication regimens.

\section{Results}

Invaluable improvements in coordinating the transition of care and in reducing hospital readmissions can be achieved by the utilization of a PCC. Utilizing the PCC, patients were seen by a provider within 7 days of hospital discharge. Medication reconciliation was performed more than $95 \%$ of patients. No-shows were reduced to an average of 2 per week. The clinic aligned with Medicare requirements and met the standards for best practice. Patients received closer follow-up with their PCP and hospital readmissions decreased. Medication reconciliation, a billable reimbursement code (CPT $111 \mathrm{~F}$ ), was utilized by providers to help maximize Medicare incentives (Figure 1). 


\section{Figure 1}

\section{Weekly Compliance with Medication Reconciliation}

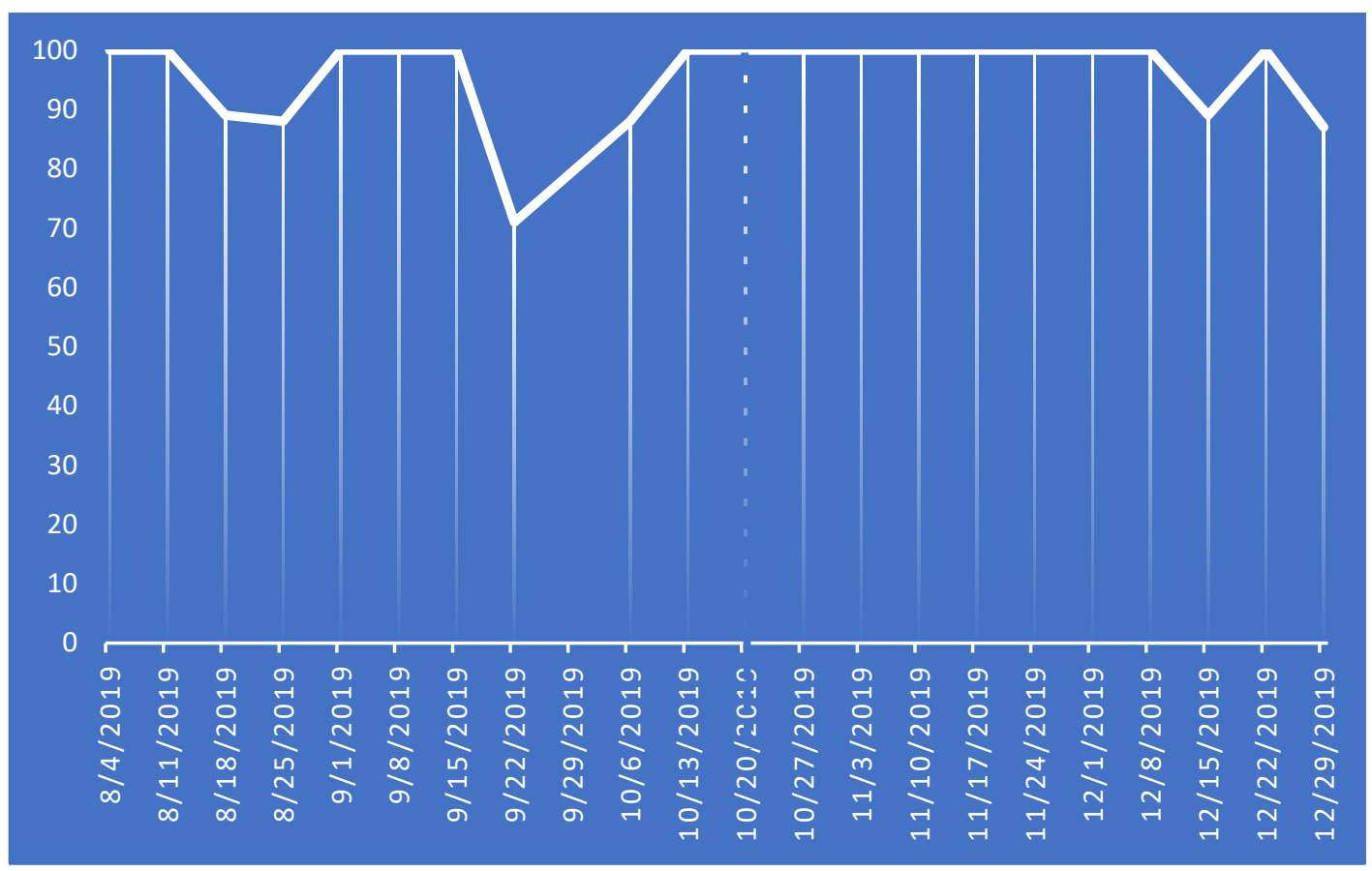

\section{Limitations}

Limitations during project implementation included inadequate staffing to cover weekend shifts. For example, the case manager worked until noon on Friday and did not return to work until Monday morning. Therefore, patients who were discharged on the weekend had no point-of-contact for scheduling an appointment with the PCC. This issue delayed contact and some patients were not contacted before their return to the emergency department. During new-hire orientation week, a noticeable decrease in medication reconciliation scores could be seen until training was reinforced.

Another limitation included the use of email between the post utilization team and the case manager. Updates and changes to appointments were discussed during meetings and necessitated extensive emails for the medical assistants to navigate in order to contact patients for appointments. The team is working on a standardized template for real-time 
updating. Lastly, there was a lapse in communication between the provider and medical assistant. Upon the patient's arrival to PCC, the medical assistant would complete the medication reconciliation; however, any provider changes to medications were not updated by the medical assistant resulting in an incomplete mediation reconciliation.

\section{Implications}

PCC is a sustainable solution that has been be implemented in three venues within the Desert Oasis Healthcare system. Replicating the PCC can also be achieved in other health care entities. Continuous quality improvements will be needed to refine processes and services; the PCC adds value to the provision of care. A job opportunity has been authorized to fill the weekend lapse of coverage. Nurse practitioners are is suitable for this role as they offer the skills to diagnosis, assess, and treat patients within their scope of practice. A new dashboard is being created to track more metrics and identify potential barriers.

\section{Conclusion}

As health care continues to evolve, connections between hospital stays and post discharge care is vital to meet the standards of best practices for patient care. Nurse practitioners can assist as providers assuring seamless handoffs for the transition of care. The PCC can meet the needs of patients, optimize post-discharge outcomes, and reduce hospital and emergency department readmissions. 


\section{References}

Baldwin, S. M. (2016). Implementing post-hospital interprofessional care team visits to improve transitions of care and decrease hospital readmission rates. https://pdfs.semanticscholar.org/ba37/1afd895a81cb759e823cef41bae87c47c9f1.p df? ga=2.43716711.311574378.1585431095-1583141909.1585431095

Dang, D., \& Dearholt, S. L. (2018). Johns Hopkins nursing evidence-based practice: Model and guidelines (3rd ed.). Sigma Theta Tau International.

Dreyer. (2014). Care transitions: Best practices and evidence-based programs. Home Healthcare Nurse, 32(5), 309-316. https://doi.org/10.1097/NHH.0000000000000069

Gilbert, J. H. V., Yan, J., \& Hoffman, S. J. (2010). A WHO Report: Framework for action on interprofessional education and collaborative practice. Journal of Allied Health, 39(3), 196-197. https://www.researchgate.net/publication/233588528_A_WHO_Report_Framewo $\underline{\text { rk_for_Action_on_Interprofessional_Education_and_Collaborative_Practice }}$

Graham, K. L., Auerbach, A. D., Schnipper, J. L., Flanders, S. A., Kim, C. S., Robinson, E. J., Ruhnke, G. W., Thomas, L. R., Kripalani, S., Vasilevskis, E. E., Fletcher, G. S., Sehgal, N. J., Lindenauer, P. K., Williams, M. V., Metlay, J. P., Davis, R. B., Yang, J., Marcantonio, E. R., \& Herzig, S. J. (2018). Preventability of early vs. late hospital readmissions in a national cohort of general medicine patients. Annals of Internal Medicine, 168(11), 766-774. https://doi.org/10.7326/M17$\underline{1724}$ 
Health Catalyst. (2018, March 23). Care transitions improvements reduces 30-day allcause readmissions saving nearly $\$ 2$ million. https://www.healthcatalyst.com/success_stories/reduce-hospital-readmissionrates-utmb/

Jackson, C., Shahsahebi, M., Wedlake, T., \& DuBard, C. A. (2015). Timeliness of outpatient follow-up: An evidence-based approach for planning after hospital discharge. Annals of Family Medicine, 13(2), 115-122. https://doi.org/10.1370/afm.1753

Low, L. L., Tay, W. Y., Tan, S. Y., Chia, E. H. S., Towle, R. M., \& Lee, K. H. (2017). Transitional home care program utilizing the integrated practice unit concept (THC-IPU): Effectiveness in improving acute hospital utilization. International Journal of Integrated Care, 17(4), 1-9. https://doi.org/10.5334/ijic.3050

Thygesen, L. C., Fokdal, S., Gjørup, T., Taylor, R. S., Zwisler, A.-D., \& Prevention of Early Readmission Research Group. (2015). Can municipality-based postdischarge follow-up visits including a general practitioner reduce early readmission among the fragile elderly $(65+$ years old $)$ ? A randomized controlled trial. Scandinavian Journal of Primary Health Care, 33(2), 65-73.

https://doi.org/10.3109/02813432.2015.1041831 